\title{
Layering Transition in Superfluid Helium Adsorbed on a Carbon Nanotube Mechanical Resonator
}

\author{
Adrien Noury, ${ }^{1, *}$ Jorge Vergara-Cruz, ${ }^{1}$ Pascal Morfin, ${ }^{2}$ Bernard Plaçais, ${ }^{2}$ Maria C. Gordillo, ${ }^{3}$ \\ Jordi Boronat, ${ }^{4}$ Sébastien Balibar, ${ }^{2}$ and Adrian Bachtold ${ }^{1}$ \\ ${ }^{1}$ ICFO_Institut De Ciencies Fotoniques, The Barcelona Institute of Science \\ and Technology Mediterranean Technology Park, 08860 Castelldefels (Barcelona), Spain \\ ${ }^{2}$ Laboratoire de Physique de l'École normale supérieure, ENS, Université PSL, CNRS, Sorbonne Université, \\ Université Paris-Diderot, Sorbonne Paris Cité, 75231 Paris Cedex 05, France \\ ${ }^{3}$ Departamento de Sistemas Físicos, Químicos y Naturales, Universidad Pablo de Olavide Carretera de Utrera, \\ km 1, E-41013 Sevilla, Spain \\ ${ }^{4}$ Departament de Física, Universitat Politècnica de Catalunya, B4-B5 Campus Nord, 08034 Barcelona, Spain
}

(Received 17 September 2018; published 23 April 2019)

\begin{abstract}
Helium is recognized as a model system for the study of phase transitions. Of particular interest is the superfluid phase in two dimensions. We report measurements on superfluid helium films adsorbed on the surface of a suspended carbon nanotube. We measure the mechanical vibrations of the nanotube to probe the adsorbed helium film. We demonstrate the formation of helium layers up to five atoms thickness. Upon increasing the vapor pressure, we observe layer-by-layer growth with discontinuities in both the number of adsorbed atoms and the speed of the third sound in the adsorbed film. These hitherto unobserved discontinuities point to a series of first-order layering transitions. Our results show that helium multilayers adsorbed on a nanotube are of unprecedented quality compared to previous works. They pave the way to new studies of quantized superfluid vortex dynamics on cylindrical surfaces, of the Berezinskii-KosterlitzThouless phase transition in this new geometry, and perhaps also to supersolidity in crystalline single layers as predicted in quantum Monte Carlo calculations.
\end{abstract}

DOI: 10.1103/PhysRevLett.122.165301

When exposing graphite to a helium vapor at low temperature, a helium film forms on the graphite surface. Several experiments have shown that the thickness of this film grows layer by layer as a function of the vapor pressure. These layers are one atom thick [1-5], and there are successive "layering transitions" between layers $n$ and $n+1$. Clements and co-workers [6,7] had predicted that the layering transitions are first-order transitions, meaning that the coverage should show a series of sharp discontinuities as a function of the helium pressure. However, such discontinuities could not be observed, probably because previous techniques required large surface areas, which were obtained, e.g., by chemically exfoliating graphite and by subsequently pressing the material into sheets. As a result, the substrate surface was made of small crystalline platelets of $\sim(10 \mathrm{~nm})^{2}$ area [4] and probably contained a sizable amount of defects including wedges where liquid helium could accumulate. Despite the modest quality of these surfaces, evidence for layer-by-layer growth was reported but without any visible discontinuities $[1,4,5,8,9]$.

By studying the mechanical resonance of a single wall carbon nanotube (NT) we have observed discontinuities in the adsorbed mass as a function of the injection of helium atoms and demonstrated for the first time that the layering transitions are indeed first order. A carbon nanotube is an excellent substrate, since the crystalline quality is high, and such nanotubes can be made free of adsorbed contamination [10]. Our results prove the very high quality of carbon nanotubes as potential substrates for new original studies like quantized superfluid vortex dynamics on cylindrical surfaces, or the Berezinskii-Kosterlitz-Thouless phase transition in a new geometry, perhaps also of supersolidity in crystalline single helium layers of variable density as predicted by Gordillo et al. [11].

Recent advances in nanomechanics have already allowed studies of liquids at small scales [12-16], but the study of the adsorption of superfluid helium on a single carbon nanotube is original [Fig. 1(a)]. We fabricate mechanical resonators by suspending a single NT of $3 \mathrm{~nm}$ diameter and $1.1 \mu \mathrm{m}$ length. This NT is contacted to two platinum electrodes and is capacitively coupled to a gate electrode [Fig. 1(b)]. In order to suppress any surface contamination, we grow the nanotube in the last step of the fabrication process and we anneal it by passing a large current $(6 \mu \mathrm{A})$ through it in the dilution refrigerator at $20 \mathrm{mK}$ prior to the adsorption of helium. This procedure already allowed us to grow xenon monolayer crystals that were commensurate with the nanotube lattice [10]. The nanotube vibrates as a doubly clamped string. The mechanical vibrations of the fundamental mode of the nanotube are driven capacitively 
(a)
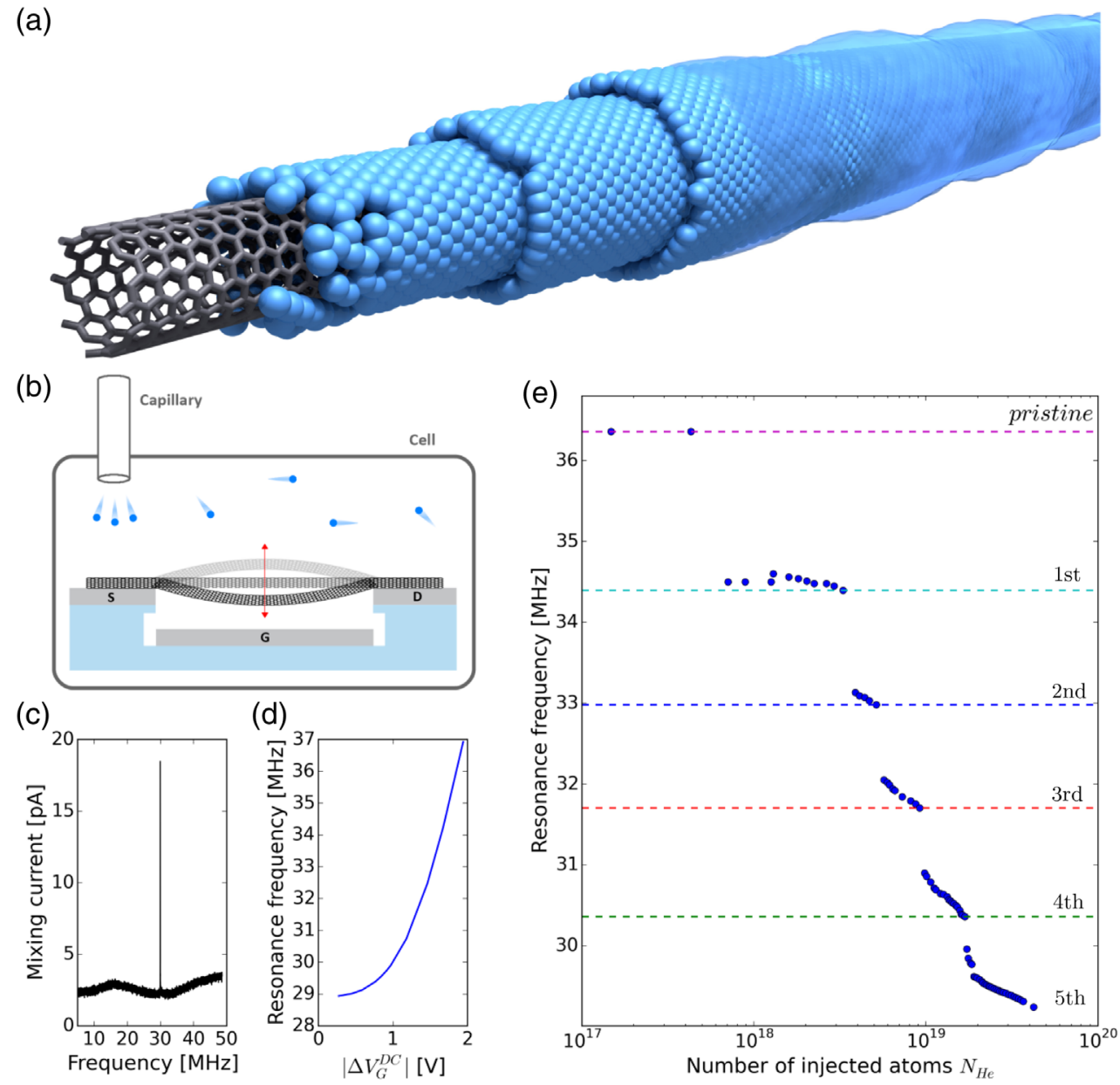

FIG. 1. Helium multilayer adsorbed on a nanotube. (a) Graphical representation of the helium multilayer on the nanotube. (b) Schematic of the nanotube mechanical resonator immersed in helium vapor. The nanotube is contacted to the platinum electrodes $S$ and $D$ and is capacitively coupled to the electrode $G$. (c) Spectrum of the mechanical vibration amplitude as a function of the drive frequency at $0.1 \mathrm{~K}$ and $V_{G}=1.908 \mathrm{~V}$. (d) Gate voltage dependence of the resonance frequency. The offset due to the work function difference between the nanotube and the gate electrode is $V_{G}=0.077 \mathrm{~V}$. (e) Layer-by-layer growth of the helium film. The resonance frequency is measured at $20 \mathrm{mK}$ as a function of the number of helium atoms injected at room temperature into the cell through the capillary. The different steps are associated to the pristine nanotube and the successive adsorption of helium layers; the steps are labeled accordingly and highlighted by the horizontal dashed lines.

and measured electrically [Fig. 1(c)] [17-19]. The resonance frequency can be tuned by a large amount around $30 \mathrm{MHz}$ when tuning the gate voltage [Figs. 1(c) and 1(d)]. The quality factor deduced from the thermal motion linewidth is $\approx 2 \times 10^{5}$. We inject ${ }^{4} \mathrm{He}$ from room temperature into the sample cell through a capillary.

A mechanical resonator based on a suspended nanotube is a remarkable sensor of atoms adsorbed on its surface [20-23]. The mass detection can reach single atom resolution [20]. Driving the resonator to detect adsorbed atoms does not affect the dynamics of the adsorbates, since the amplitude of the driven vibrations can be kept smaller than the amplitude of thermal vibrations. The resonance frequency $f_{0}=(1 / 2 \pi) \sqrt{(K / M)}$ depends on the ratio of the string elasticity, also called spring constant $K$, to the effective mass $M$. The coefficient $K=k_{\mathrm{NT}}+k_{\mathrm{He}}$ is the sum of the respective elasticities of the naked NT - also called pristineand of the adsorbed helium. Similarly, the total mass $M=$ $m_{\mathrm{NT}}+m_{\mathrm{He}}$ is the sum of the respective masses of the NT and of the adsorbed helium. With helium adsorbed, both $K$ and $M$ increase, but their contributions to the resonance frequency have opposite signs, so that we could distinguish between them by varying the gate voltage. This is a great advantage of our experiment. Previous studies of two-dimensional helium superfluids often used torsional oscillators $[4,5,24]$. Contrary to our case, these torsional oscillators were macroscopic with not much possibility to change their resonance frequency, so that mass effects were difficult to distinguish from elasticity effects [25]. As we shall see below, we have observed the two effects in two different temperature regimes.

By studying the transport properties of electrons inside the NT, we have demonstrated that our nanotubes are free 

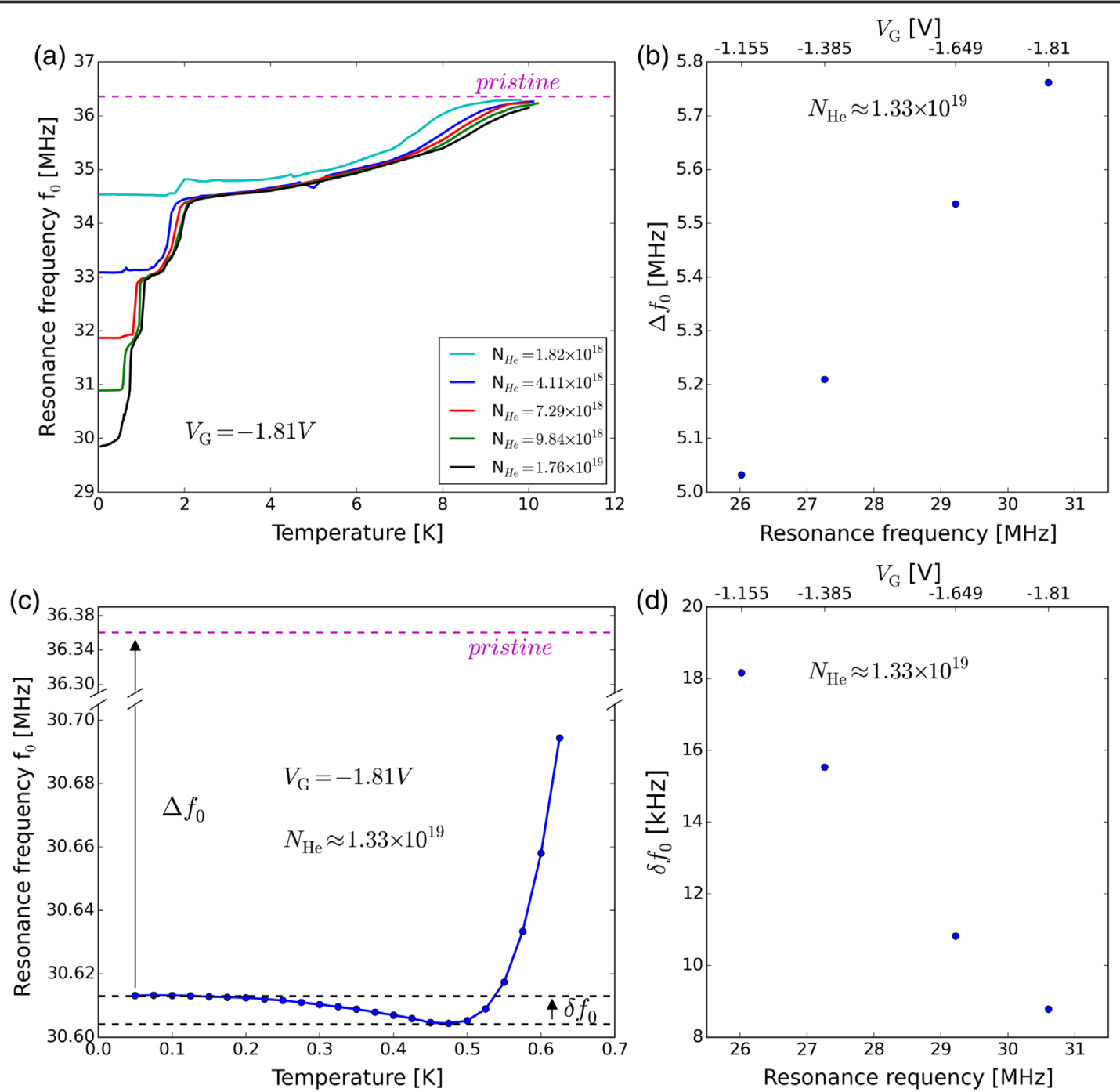

FIG. 2. Layer-by-layer desorption upon increasing temperature. (a) Resonance frequency as a function of temperature. The "pristine" is the carbon nanotube without any adsorption. (b) Frequency shift $\Delta f_{0}$ related to $m_{\mathrm{He}}$ as a function of gate voltage (upper axis) and resonance frequency (lower axis). As indicated in (c), $\Delta f_{0}$ is the change in resonance frequency between the pristine nanotube and the dressed nanotube at $20 \mathrm{mK}$. (c) Temperature dependence of the resonance frequency in the low-temperature regime. (d) Frequency shift $\delta f_{0}$ related to $k_{\mathrm{He}}$ as a function of gate voltage (upper axis) and resonance frequency (lower axis). The shift $\delta f_{0}$ is represented in (c).

of disorder. As a function of the gate voltage, the conductance is periodically modulated due to quantum electron interference [26]. This periodic modulation would be deteriorated by a tiny amount of disorder (see Sec. I of Supplemental Material [27]).

We measured the nanotube length $L \simeq 1.1 \mu \mathrm{m}$ from the characteristic source-drain voltage $V_{C}=1.5 \mathrm{mV}$ of the electron interference pattern. This length is $L=h v_{F} /$ $2 e V_{C}$ [26], where $v_{F}=8 \times 10^{5} \mathrm{~m} / \mathrm{s}$ is the Fermi velocity of nanotubes. It is consistent with the width of the trench, and we obtained a similar but less reliable length $(L \approx 1 \mu \mathrm{m})$ from the Coulomb blockade measurements of the conductance.

We observe a series of steps when measuring the resonance frequency of the nanotube as a function of the number $N_{\mathrm{He}}$ of helium atoms injected into the sample chamber [Fig. 1(e)]. We proceed by successive injections of $\approx 76 \mathrm{~cm}^{3}$ of helium gas at a pressure set between 0.08 and 3 mbar. When we increase $N_{\mathrm{He}}$, the vapor pressure $P$ in the surrounding of the nanotube gets larger, but $P$ cannot be quantitatively estimated due to the unknown adsorption of helium atoms onto the cell walls. We attribute the reduction of the resonance frequency $\Delta f_{0}$ measured in Fig. 1(e) to the helium mass $m_{\mathrm{He}}$ adsorbed on the NT. Each plateau in $f_{0}$ is assigned to a different layer adsorbed on the nanotube. At the completion of each layer, a new layer starts and the resonance frequency jumps down. It means a divergence of the coverage at constant helium pressure, that is, a divergence of the compressibility and a vanishing of the sound speed in the helium layer. This is in agreement with the first-order transition predicted for the layering transition [6]. The helium density at each discontinuity 

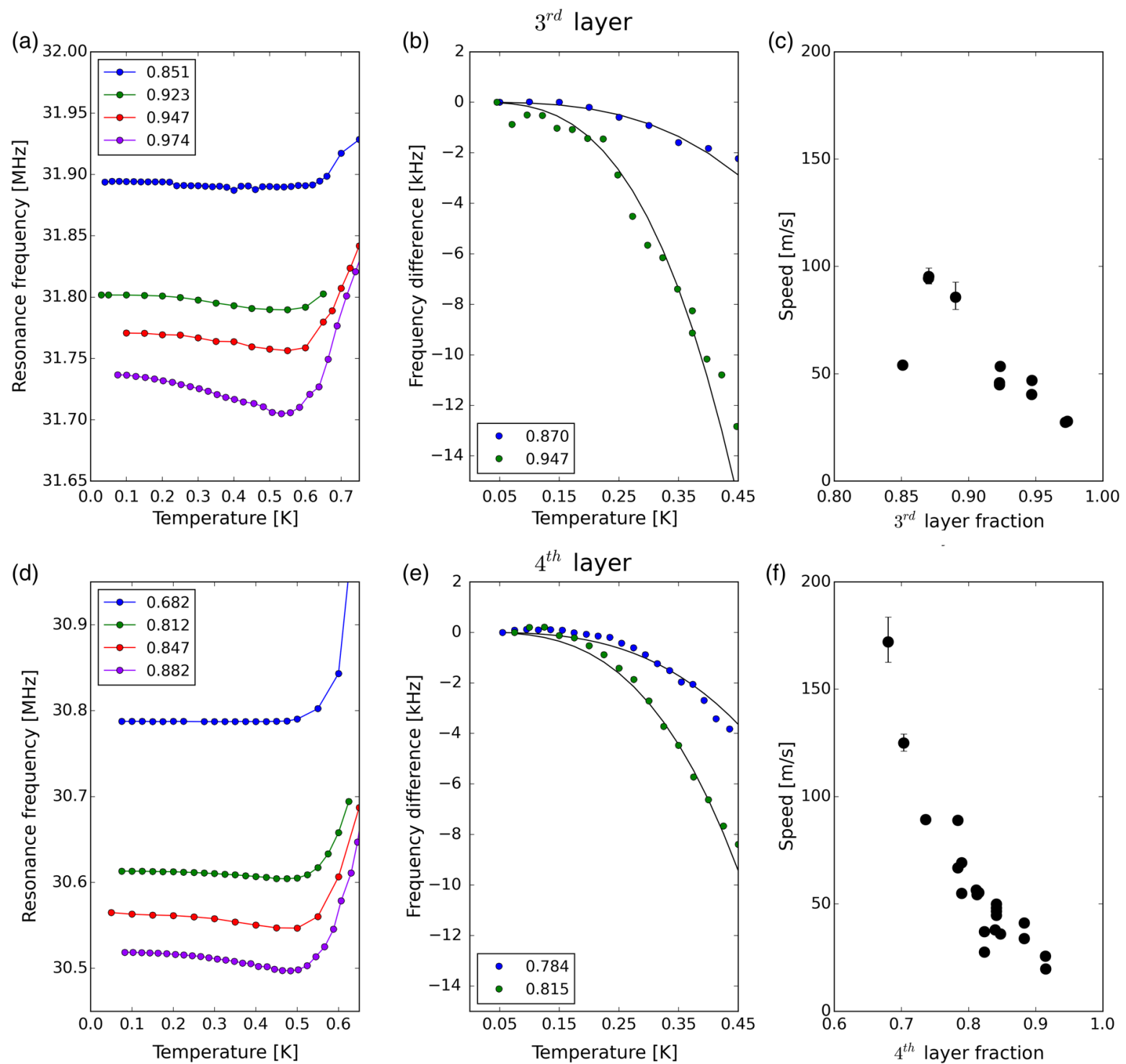

FIG. 3. Mechanical sensing of the third sound. (a) Resonance frequency as a function of temperature in the low-temperature regime for the third layer. The different curves correspond to different layer filling fractions, which are indicated in the legend. (b) Measured temperature dependence of the resonance shift (dots). The lines are cubic temperature dependencies, as expected from Eq. (1). (c) Third sound speed as a function of the layer filling fraction of the third layer. The error bars are obtained from the comparison between the measured $\delta f_{0}(T)$ dependence and Eq. (1). (d)-(f) Same as (a)-(c) but for the fourth layer.

agrees within $6 \%$ with the established density of helium adsorbed on graphite (Sec. III of Supplemental Material [27]). During the completion of each layer, helium atoms must be homogeneously distributed over the surface and the chemical potential of the helium vapor needs to increase to equilibrate the increase of the repulsion between atoms. This explains the observed finite slope $\partial f_{0} / \partial N_{\mathrm{He}}$ of the plateaus [Fig. 1(e)].

Figure 2(a) shows the layer-by-layer desorption of helium from the surface of the nanotube, but now as a function of temperature up to $10 \mathrm{~K}$ and for five successive amounts of helium in the cell. The frequency of the steps is similar to those measured while varying $N_{\mathrm{He}}$ at constant temperature [Fig. 1(e)]. One temperature sweep takes a few days to ensure the reproducibility of measurements. The layering transitions are rounded, probably because the layering transitions have a critical point of order $1 \mathrm{~K}$ (see Ref. [33]). By varying the gate voltage, consequently the spring constant, we have confirmed that the variation of the resonance frequency $f_{0}$ in Fig. 2(a) is due to the change in the resonator mass. Indeed, the large frequency change $\Delta f_{0}$ is an increasing function of $f_{0}$ [Fig. 2(b) and Sec. IV of Supplemental Material [27] ].

The first and second layers show some peculiar structures which would need further study to be compared with predictions by Boronat and co-workers (see Ref. [34] and Sec. VI of Supplemental Material [27]). We thus focus on the third and fourth layers. By looking more precisely at the frequency variation below $0.7 \mathrm{~K}$, we discovered the 
existence of a small minimum near $0.5 \mathrm{~K}$ [see Fig. 2(c)]. The rise of $f_{0}$ above $\sim 0.5 \mathrm{~K}$ in Fig. 2(c) signals the desorption of helium atoms from the nanotube. Figure 2(d) shows the dependence of the depth $\delta f_{0}$ of this small minimum on the gate voltage. Contrary to the case of the large frequency shift $\Delta f_{0}$ of Fig. 2(b), the slope is negative [see Fig. 2(d) and Sec. IV of Supplemental Material [27] ]. It means that this small minimum is due to the helium contribution to the total spring constant of the resonator.

We attribute it to the temperature variation of the helium surface tension, that is, to the growing entropy of the helium film [35]. It is due to the third sound states in the case of thin superfluid films [36]. These waves are predicted to be longitudinal with no sizable displacement of helium atoms in the direction perpendicular to the surface, except for densities near the layering transition [37]. The third sound has a linear energy dispersion given by the speed $c$. The resulting dependence of $\delta f_{0}$ on temperature $T$ is

$$
\delta f_{0}(T)=-0.074 \frac{1}{m_{\mathrm{NT}} f_{0}} \frac{r_{\mathrm{He}}}{L} \frac{\left(k_{B} T\right)^{3}}{(\hbar c)^{2}},
$$

where $m_{\mathrm{NT}}$ is the mass of the pristine nanotube, $L$ the suspended nanotube length, $k_{B}$ the Boltzmann constant, and $\hbar$ the reduced Planck constant. See Sec. V of the Supplemental Material for a detailed derivation [27].

Figures 3(b) and 3(e) show a very good agreement with the $T^{3}$ law of Eq. (1) from which we could extract the sound velocity $c$ [Figs. 3(c) and 3(f)]. The layer fractions are computed by comparing the resonance frequency at $20 \mathrm{mK}$ to the frequency of the jump indicated by the dashed line in Fig. 1(e). We notice a large jump in sound velocity from the end of the third layer $(\sim 30 \mathrm{~m} / \mathrm{s})$ to the beginning of the fourth layer $(\sim 170 \mathrm{~m} / \mathrm{s})$, in agreement with the predictions of a first-order transition by Clements and co-workers [6,7]. Although we found no evidence of ripplon excitations in our system, studying thicker helium layers may lead to their observation in future work.

Our work shows that helium layers adsorbed on carbon nanotubes are of unprecedented quality. This system is of great interest for the study of different quantum phenomena. The cylindrical boundary condition of the superfluid imposes quantized translational velocities of a vortex around the circumference [38]. This boundary condition also modifies the vortex-vortex interaction energy [38], so that it might alter the Berezinskii-Kosterlitz-Thouless topological phase transition [39]. According to Gordillo et al. [11], helium monolayers adsorbed on nanotubes may become supersolid at low temperature. In summary, carbon nanotubes could be used for original studies of helium-based quantum phenomena.

We thank N. Guenther, P. Massignan, A. Fetter, and G. Williams for fruitful discussions. This work is supported by the ERC advanced Grant No. 692876, the Foundation Cellex, the CERCA Programme, AGAUR, Severo Ochoa
(SEV-2015-0522), the Grant No. FIS2015-69831-P of MINECO, and the Fondo Europeo de Desarrollo Regional (FEDER). M. C. G. acknowledges partial financial support from the MINECO (Spanish Ministry of Economy) Grant No. FIS2017-84114-C2-2-P. M. C. G. also acknowledges the use of the C3UPO computer facilities at the Universidad Pablo de Olavide. J. B. acknowledges financial support from MINECO Grants No. FIS2014-56257-C2-1-P and No. FIS2017-84114-C2-1-P.

*Present address: Laboratoire Charles Coulomb (L2C), Univ Montpellier, CNRS, Montpellier, France.

[1] G. Zimmerli, G. Mistura, and M. H. W. Chan, Phys. Rev. Lett. 68, 60 (1992).

[2] D. S. Greywall and P. A. Busch, Phys. Rev. Lett. 67, 3535 (1991).

[3] D. S. Greywall, Phys. Rev. B 47, 309 (1993).

[4] P. A. Crowell and J. D. Reppy, Phys. Rev. B 53, 2701 (1996).

[5] J. Nyéki, A. Phillis, A. Ho, D. Lee, P. Coleman, J. Parpia, B. Cowan, and J. Saunders, Nat. Phys. 13, 455 (2017).

[6] W. M. Saslow, G. Agnolet, C. E. Campbell, B. E. Clements, and E. Krotscheck, Phys. Rev. B 54, 6532 (1996).

[7] B. E. Clements, E. Krotscheck, and H. J. Lauter, Phys. Rev. Lett. 70, 1287 (1993).

[8] H. Godfrin and H.-J. Lauter, Prog. Low Temp. Phys. 14, 213 (1995).

[9] E. Menachekanian, V. Iaia, M. Fan, J. Chen, C. Hu, V. Mittal, G. Liu, R. Reyes, F. Wen, and G. A. Williams, Phys. Rev. B 99, 064503 (2019).

[10] A. Tavernarakis, J. Chaste, A. Eichler, G. Ceballos, M. C. Gordillo, J. Boronat, and A. Bachtold, Phys. Rev. Lett. 112, 196103 (2014).

[11] M. C. Gordillo, C. Cazorla, and J. Boronat, Phys. Rev. B 83, 121406 (2011).

[12] E. Gil-Santos, C. Baker, D. T. Nguyen, W. Hease, C. Gomez, and A. Lemaître, S. Ducci, G. Leo, and I. Favero, Nat. Nanotechnol. 10, 810 (2015).

[13] A. D. Kashkanova, A. B. Shkarin, C. D. Brown, N.E. Flowers-Jacobs, L. Childress, S. W. Hoch, L. Hohmann, K. Ott, J. Reichel, and J. G. E. Harris, Nat. Phys. 13, 74 (2017).

[14] S. Kaminski, L. L. Martin, S. Maayani, and T. Carmon, Nat. Photonics 10, 758 (2016).

[15] G. I. Harris, D. L. McAuslan, E. Sheridan, Y. Sachkou, C. Baker, and W. P. Bowen, Nat. Phys. 12, 788 (2016).

[16] D. L. McAuslan, G. I. Harris, C. Baker, Y. Sachkou, X. He, E. Sheridan, and W. P. Bowen, Phys. Rev. X 6, 021012 (2016).

[17] V. Sazonova, Y. Yaish, H. Üstünel, D. Roundy, T. A. Arias, and P. L. McEuen, Nature (London) 431, 284 (2004).

[18] J. Moser, A. Eichler, J. Güttinger, M. I. Dykman, and A. Bachtold, Nat. Nanotechnol. 9, 1007 (2014).

[19] S. L. de Bonis, C. Urgell, W. Yang, C. Samanta, A. Noury, J. Vergara-Cruz, Q. Dong, Y. Jin, and A. Bachtold, Nano Lett. 18, 5324 (2018).

[20] J. Chaste, A. Eichler, J. Moser, G. Ceballos, R. Rurali, and A. Bachtold, Nat. Nanotechnol. 7, 301 (2012).

[21] H.-Y. Chiu, P. Hung, H. W. Ch. Postma, and M. Bockrath, Nano Lett. 8, 4342 (2008). 
[22] Z. Wang, J. Wei, P. Morse, J. G. Dash, O. E. Vilches, and D. H. Cobden, Science 327, 552 (2010).

[23] H.-C. Lee, O. E. Vilches, Z. Wang, E. Fredrickson, P. Morse, R. Roy, B. Dzyubenko, and D. H. Cobden, J. Low Temp. Phys. 169, 338 (2012).

[24] D. J. Bishop and J. D. Reppy, Phys. Rev. Lett. 40, 1727 (1978).

[25] X. Mi and J. D. Reppy, J. Low Temp. Phys. 175, 104 (2014).

[26] W. Liang, M. Bockrath, D. Bozovic, J. H. Hafner, M. Tinkham, and H. Park, Nature (London) 411, 665 (2001).

[27] See Supplemental Material at http://link.aps.org/ supplemental/10.1103/PhysRevLett.122.165301 for the description of quantum electron transport measurements, the detection of mechanical vibrations, the measured areal density of different layers, the derivation of Eq. (1), and theoretical calculations on the first two helium layers, which includes Refs. [28-32].

[28] E. A. Laird, F. Kuemmeth, G. A. Steele, K. Grove-Rasmussen, J. Nygard, K. Flensberg, and L. P. Kouwenhoven, Rev. Mod. Phys. 87, 703 (2015).
[29] P. Corboz, M. Boninsegni, L. Pollet, and M. Troyer, Phys. Rev. B 78, 245414 (2008).

[30] K. R. Atkins, Can. J. Phys. 31, 1165 (1953).

[31] J. E. Rutledge, W. L. McMillan, J. M. Mochel, and T. E. Washburn, Phys. Rev. B 18, 2155 (1978).

[32] M. C. Gordillo and D. M. Ceperley, Phys. Rev. B 58, 6447 (1998).

[33] S. Ramesh, Q. Zhang, G. Torzo, and J. D. Maynard, Phys. Rev. Lett. 52, 2375 (1984).

[34] M. C. Gordillo and J. Boronat, Phys. Rev. B 86, 165409 (2012).

[35] K. R. Atkins and Y. Narahara, Phys. Rev. 138, A437 (1965).

[36] K. R. Atkins, Phys. Rev. 113, 962 (1959).

[37] B. E. Clements, H. Forbert, E. Krotscheck, H. J. Lauter, M. Saarela, and C. J. Tymczak, Phys. Rev. B 50, 6958 (1994).

[38] N.-E. Guenther, P. Massignan, and A. L. Fetter, Phys. Rev. A 96, 063608 (2017).

[39] J. Machta and R. A. Guyer, J. Low Temp. Phys. 74, 231 (1989). 